\title{
Wafer Scale Synthesis of Dense Aligned Arrays of Single-Walled Carbon Nanotubes
}

\author{
Weiwei Zhou, Christopher Rutherglen, and Peter J. Burke ( $\square)$ \\ Department of Electrical Engineering and Computer Science, University of California, Irvine, CA 92697, USA \\ Received: 1 May 2008/Revised: 7 June 2008/Accepted: 7 June 2008 \\ CTsinghua Press and Springer-Verlag 2008
}

\begin{abstract}
Here we present an easy one-step approach to pattern uniform catalyst lines for the growth of dense, aligned parallel arrays of single-walled carbon nanotubes (SWNTs) on quartz wafers by using photolithography or polydimethylsiloxane (PDMS) stamp microcontact printing $(\mu \mathrm{CP})$. By directly doping an $\mathrm{FeCl}_{3} /$ methanol solution into Shipley 1827 photoresist or polyvinylpyrrolidone (PVP), various catalyst lines can be wellpatterned on a wafer scale. In addition, during the chemical vapor deposition (CVD) growth of SWNTs the polymer layers play a very important role in the formation of mono-dispersed nanoparticles. This universal and efficient method for the patterning growth of SWNTs arrays on a surface is compatible with the microelectronics industry, thus enabling of the fabrication highly integrated circuits of SWNTs.
\end{abstract}

\section{KEYWORDS}

Single-walled carbon nanotubes (SWNTs), chemical vapor deposition (CVD), photolithography, microcontact printing $(\mu \mathrm{CP})$

\section{Introduction}

Single-walled carbon nanotubes (SWNTs) as a typical one-dimensional nanomaterial promise to be one of the outstanding candidates for state-ofthe-art electronic devices in the silicon industry of the future [1-5]. Controlled synthesis of aligned SWNTs arrays on substrates is useful for application in highly integrated caborn nanotube-based circuits [6-10] and especially high frequency electronics [11-17] where impedance matching is a necessity. Advances in the growth of horizontally aligned SWNTs arrays with very high densities have recently been achieved on single-crystal quartz wafers by chemical vapor deposition (CVD) of ethanol [18,
19]. For synthesis of aligned arrays of SWNTs on substrates, pre-designed catalyst structures and immobilizing of catalysts are both very important. Especially on quartz wafers Rogers' group found that if catalysts are not patterned on the surface, a large number of curved or random SWNTs are produced by the CVD growth [20]. Thus, an easy strategy to build catalyst structures on the surface of wafers is an important goal in the area of SWNT growth $[3,7,18,21-24]$. In this work, we introduce an easy one-step method to pattern catalyst lines on quartz wafers and successfully obtain patterned, dense, and high-quality SWNT arrays on quartz wafers by CVD of methane. We directly doped $\mathrm{FeCl}_{3}$ into Shipley 1827 photoresist or polyvinglpyrrolidone (PVP)

Address correspondence to pburke@uci.edu 
in methanol solution as the catalytic precursors. Due to the presence of the polymers we can design perfect patterns of catalysts on a wafer scale using only a simple photolithography technique or the polydimethylsiloxane (PDMS) stamp microcontact printing $(\mu \mathrm{CP})$ technique. Moreover, we found both Shipley 1827 photoresist and PVP to be effective in contributing to the formation of mono-dispersed catalyst nanoparticles as well as hindering them from aggregating on the substrate during the CVD process. Especially for the former, very uniform and almost perfectly aligned arrays of SWNTs were synthesized with an average density of $\sim 10$ SWNTs / $\mu \mathrm{m}$ and lengths of up to a millimeter.

\section{Experimental}

\subsection{Photolithography}

ST-cut quartz wafers (Hoffman Materials Inc.) were annealed at $900{ }^{\circ} \mathrm{C}$ in air for $1 \mathrm{~h}$ before a standard UV photolithography process was applied. Shipley 1827 positive photoresist (Shipley Company) was doped with $5 \mathrm{mmol} / \mathrm{L} \mathrm{FeCl}_{3} /$ methanol solution and was then used to pattern lines with $20 \mu \mathrm{m}$ width, 2 $\mathrm{cm}$ length, and spacings gradually increasing from $10 \mu \mathrm{m}$ to $100 \mu \mathrm{m}$ on quartz wafers. This forms the catalytic precursors for subsequent catalysis of the growth of SWNTs. The lines on the substrates must be perpendicular to the $X$ axis of the quartz surface according to a previous report [18].

\subsection{Microcontact printing}

Before a typical printing process, a PDMS stamp with regular lines was treated by $\mathrm{O}_{2}$ plasma for $5 \mathrm{~min}$ in order to improve the hydrophilicity of the surface. A fresh methanol solution of $10 \mathrm{mmol} / \mathrm{L} \mathrm{FeCl}_{3}$ and PVP with $20-40 \mathrm{mmol} / \mathrm{L}$ concentration (expressed per mole of monomer) was prepared and dipped onto the surface of the stamp. After drying in air, the ink was transferred onto the surface of a clean quartz wafer without annealing by making direct contact between the stamp and the substrate for $2 \mathrm{~min}$. The ink lines on the substrates also must be perpendicular to the $X$ axis of the quartz surface.

\subsection{Growth of SWNTs arrays}

After photolithography or $\mu \mathrm{CP}$, the wafers were treated by $\mathrm{O}_{2}$ plasma for $15 \mathrm{~min}$ or calcined at $700{ }^{\circ} \mathrm{C}$ in air for $5 \mathrm{~min}$ to remove the polymer layer and form $\mathrm{Fe}_{x} \mathrm{O}_{y}$ nanoparticles. For the case of $\mathrm{FeCl}_{3} /$ Shipley 1827 photoresist as the catalytic precursor, the substrate was put into a horizontal furnace (Lindberg 3 in. diameter tube furnace) and heated to $920{ }^{\circ} \mathrm{C}$ under the protection of $\mathrm{Ar}$ (1500 standard cubic centimeters per minute (sccm)). Subsequently, a flow of $\mathrm{CH}_{4} / \mathrm{H}_{2}$ gas mixture (1100 sccm/220 sccm) was introduced for SWNT growth. After $30 \mathrm{~min}$, the furnace was cooled down to room temperature in Ar (1500 sccm). When $\mathrm{FeCl}_{3} / \mathrm{PVP}$ was used as the catalytic precursor, before the CVD growth of SWNTs it was necessary for reduction to effected by introduction of $\mathrm{H}_{2} / \mathrm{Ar}$ (220 sccm/1000 sccm) for 5-15 min depending on the concentration of PVP used. Scanning electron microscopy (SEM, Hitachi S-4700-2 FESEM, operated at $1 \mathrm{kV}$ acceleration voltage), atomic force microscopy (AFM, Vecco Nanoscope IIIa, operated in tapping mode) and micro-Raman spectroscopy (JY LabRam ARAMIS, with an excitation laser wavelength of 633 $\mathrm{nm}$ ) were used to characterize the arrays of SWNTs on quartz wafers formed by CVD growth.

\section{Results}

\subsection{Shipley 1827 photoresist as the polymer layer}

The strategy is shown in Scheme 1. SEM images of typical arrays of SWNTs on quartz wafers obtained by using solution of $5 \mathrm{mmol} / \mathrm{L} \mathrm{FeCl}_{3}$ and Shipley 1827 photoresist as catalytic precursors and simple photolithography to pattern the uniform catalyst lines are shown in Fig. 1. The arrays of SWNTs are perfectly aligned and have very uniform density of $\sim 10 \mathrm{SWNTs} / \mu \mathrm{m}$ on a $25 \mathrm{~mm} \times 40 \mathrm{~mm}$ wafer without any curved or random SWNTs, even though the size of the spacings between catalyst lines on the substrates gradually increased from $10 \mu \mathrm{m}$ to $100 \mu \mathrm{m}$. Only in the area of the catalyst lines themselves are some short curved tubes visible. Orientation of the SWNTs was observed along the $X$-axis direction in 


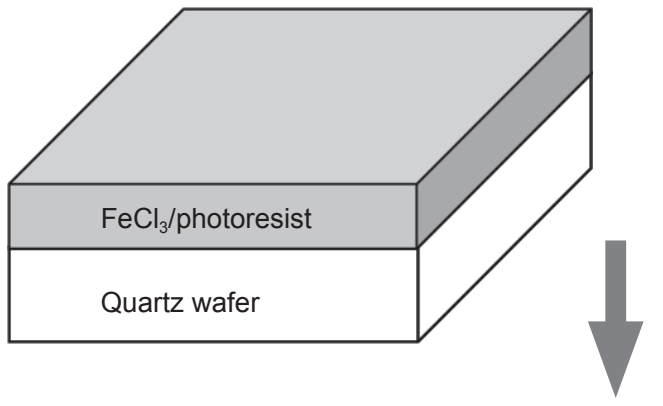

$\mathrm{FeCl}_{3} /$ photoresist lines

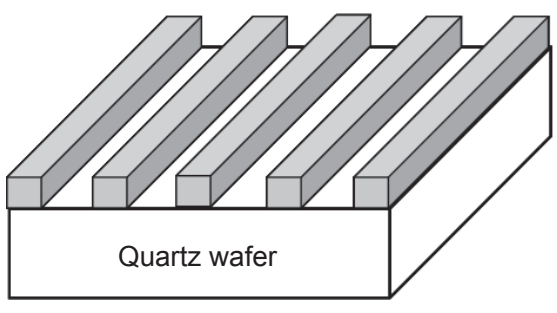

$\mathrm{Fe}_{x} \mathrm{O}_{y}$ nanoparticles lines

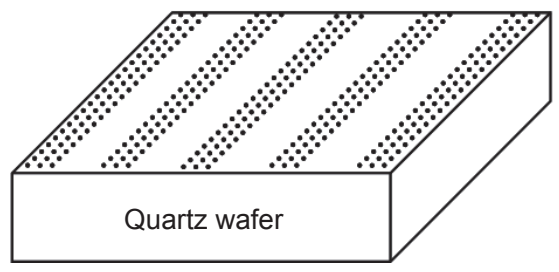

SWNTs arrays

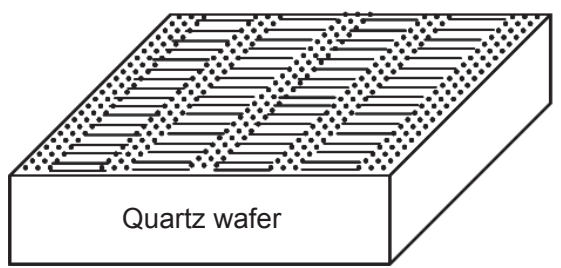

Scheme 1 Schematic illustration of the procedures for patterning catalyst lines by simple photolithography and the growth of arrays of SWNTs on quartz wafers
Step 1 A standard photolithography process

Step 2 Treated by $\mathrm{O}_{2}$ plasma for $15 \mathrm{~min}$ or calcined at $700{ }^{\circ} \mathrm{C}$ in air for $5 \mathrm{~min}$

Step 3 Growth of arrays of SWNTS by CVD of methane accordance with previous reports [18]. The results are reproducible because the standard photolithography process ensures that the uniform catalyst lines are well-patterned on a large wafer scale. Thermal annealing of the substrates in air is necessary before photolithography because thermal treatment of quartz wafers at high temperature greatly decreases the number of $-\mathrm{OH}$ groups on the surface [25] and thus improves the adhesion between photoresist and quartz wafers. Although thermal annealing for a long time at high temperature can increase the number of the atomic steps on the surface of quartz

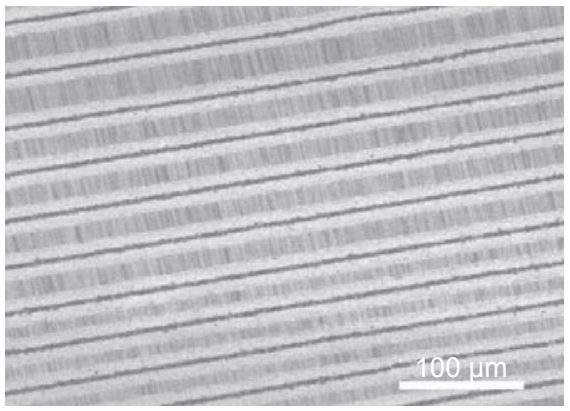

(a)

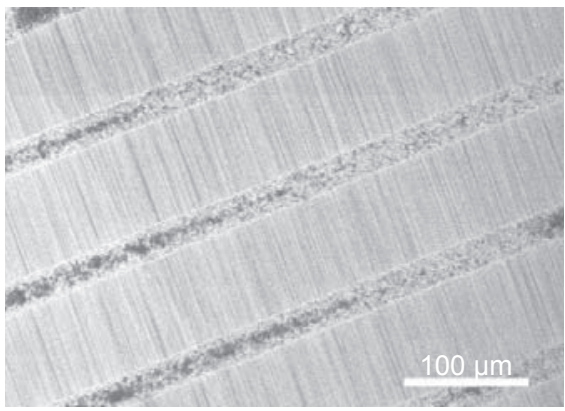

(b)

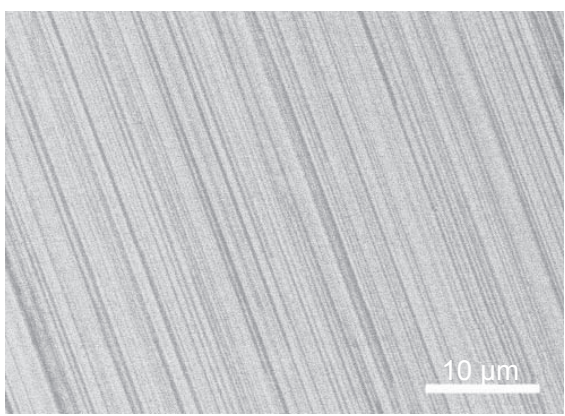

(c)

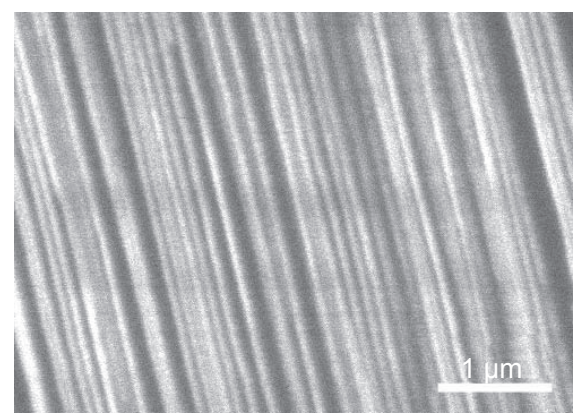

(d)

Figure 1 SEM images of large-scale perfect aligned arrays of SWNTs grown along the $X$ axis of ST-cut quartz wafers by using $\mathrm{FeCl}_{3} /$ Shipley 1827 photoresist as catalytic precursors and a simple photolithography technique to pattern the uniform catalyst lines. Low-magnification SEM images (a) and (b) correspond to different areas with narrow gaps and with wide gaps between catalyst lines, respectively. The dark lines are catalyst lines where there are some random SWNTs. Higher-magnification SEM images (c) and (d) show aligned arrays of SWNTs between catalyst lines. The density of this array of SWNTs is $\sim 10$ SWNTs/ $\mu \mathrm{m}$ according to (d) 
[6] or sapphire wafers [26], in our observations by AFM (Fig. 2(b)), there were no obvious atomic steps produced on the quartz surface by 1 -h thermal treatment. This result also proves that in our experiments SWNTs are mainly guided by the direction of the lattice, not by the atomic steps on the surface. This leads us to believe that, contrary to reports by Rogers' group [6, 7, 18], but in agreement with reports by Liu's group [19], dense aligned arrays of SWNTs can be obtained without long-time thermal annealing of quartz wafers before the CVD process.

Although a large quantity of photoresist was used in this process, no contamination of the wafer or SWNTs was observed nor did it disturb the growth of SWNTs. In the AFM image (Fig. 2(b)), the SWNTs were found to be very clean and straight on the substrates with no amorphous carbon or catalyst nanoparticles observed. According to Fig. 2(a), the lengths of most SWNTs exceed $200 \mu \mathrm{m}$ and a few reach $\sim 1 \mathrm{~mm}$ in length. By using AFM to measure 150 SWNTs we calculated that the distribution of diameters was $1.1 \mathrm{~nm} \pm 0.4 \mathrm{~nm}$, indicating that almost all of the tubes are individual single-walled tubes and the arrays have a very narrow distribution of diameters. Figure 2(c) shows a typical Raman spectrum of the semiconducting tubes with a radial breathing mode (RBM) peak at $156 \mathrm{~cm}^{-1}$ [27]. The RBM signals of metallic tubes are masked by the broad peak at $\sim 205 \mathrm{~cm}^{-1}$ arising from single-crystal quartz wafers [27].

\subsection{PVP as the polymer layer}

Besides using the photolithography method to realize the patterned growth of arrays of SWNTs on quartz wafers, the PDMS stamp $\mu \mathrm{CP}$ technique with the assistance of PVP can also be employed to pattern

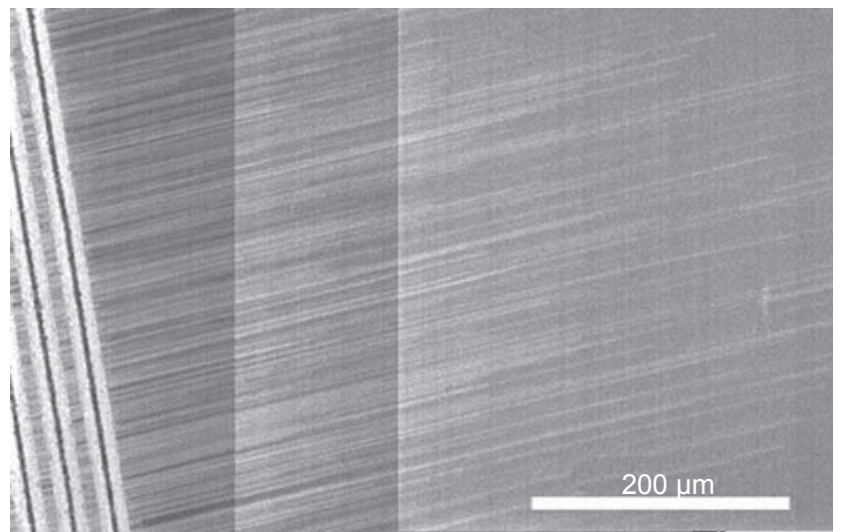

(a)

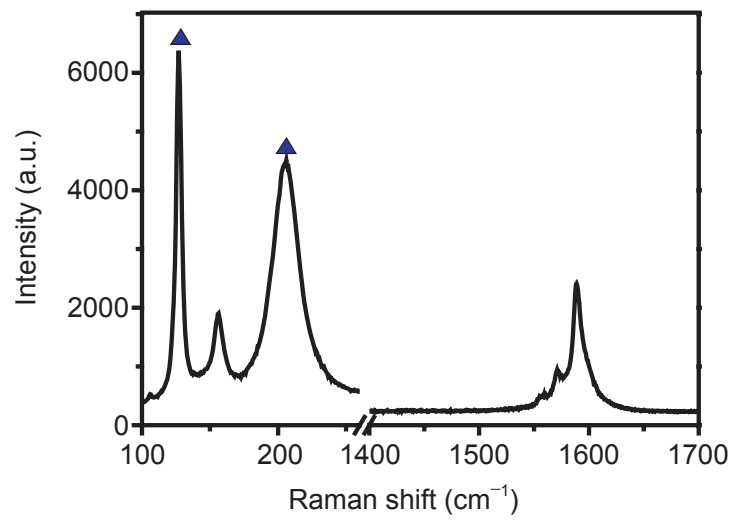

(c)

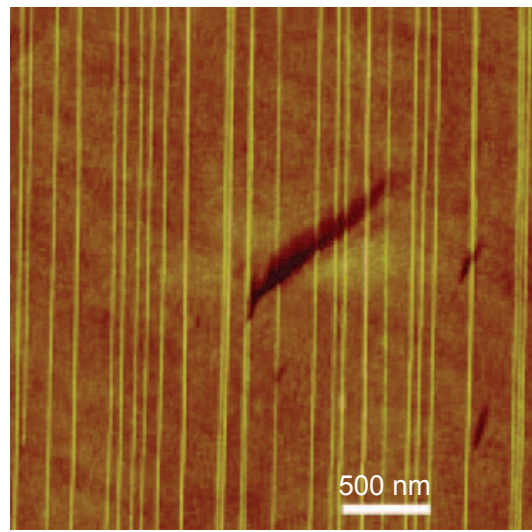

(b)

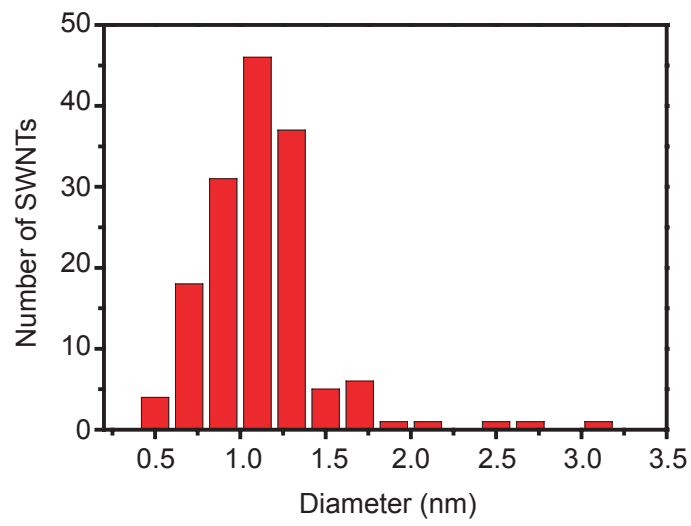

(d)

Figure 2 (a) SEM image of SWNT arrays grown from the edges of catalysts by using $\mathrm{FeCl}_{3} /$ Shipley 1827 photoresist as catalytic precursors. The lengths of most SWNTs are more than $200 \mu \mathrm{m}$ and a few reach $\sim 1 \mathrm{~mm}$ in length. (b) AFM image of the sample in Fig. 1, and the corresponding Raman spectrum (c). The excitation wavelength of the micro-Raman laser is 633 $\mathrm{nm}$. The peaks marked by blue triangles in the Raman spectrum arise from the single-crystal quartz substrate. (d) Distribution of diameters of SWNTs arrays obtained by measuring 150 SWNTs. The average diameter of the SWNTs is $\sim 1.1 \mathrm{~nm}$ 
catalyst lines on quartz wafers. In this case, there is no need for thermal annealing of the substrates before printing the catalyst. Figure 3 shows the results of SWNT growth on quartz wafers using $\mathrm{FeCl}_{3} / \mathrm{PVP}$ as catalytic precursors and the PDMS $\mu \mathrm{CP}$ technique to pattern the catalyst lines. The concentration of $\mathrm{FeCl}_{3}$ was $10 \mathrm{mmol} / \mathrm{L}$ and the concentration of PVP was 40 $\mathrm{mmol} / \mathrm{L}$ (expressed per mole of monomer). In the SEM images shown in Figs. 3(a)-(c), the catalyst lines were successfully patterned on a large scale and aligned arrays of SWNTs with a density of 2 5 SWNTs / $\mu \mathrm{m}$

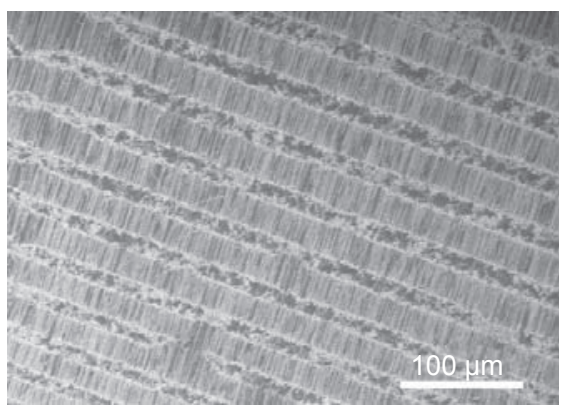

(a)

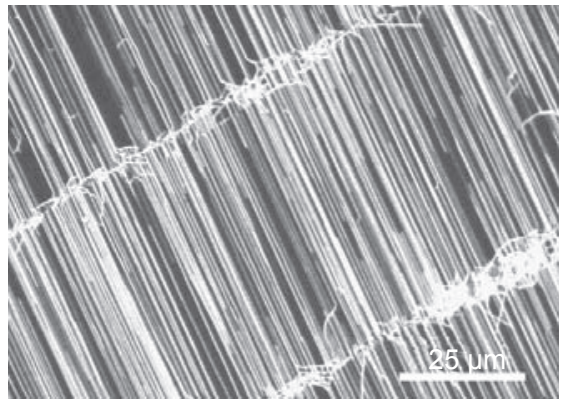

(c)

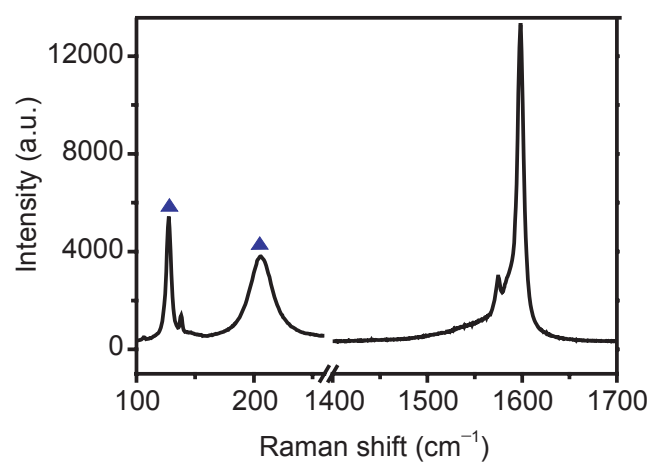

(e)

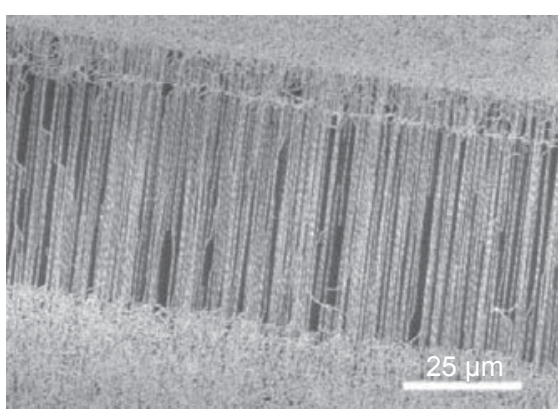

(b)

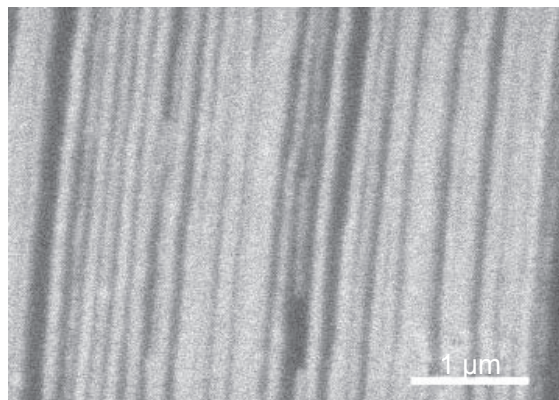

(d)

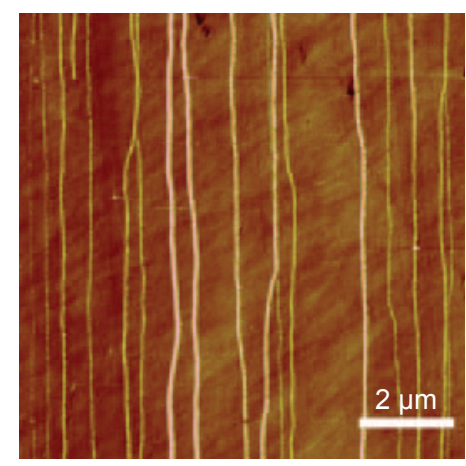

(f)
Figure 3 SEM images ((a)-(d)) of the arrays of SWNTs grown on quartz wafers by using $\mathrm{FeCl}_{3} / \mathrm{PVP}$ as catalytic precursors and the PDMS $\mu \mathrm{CP}$ technique to pattern the catalyst lines. The concentration of $\mathrm{FeCl}_{3}$ is $10 \mathrm{mmol} / \mathrm{L}$ and the concentration of PVP is $40 \mathrm{mmol} / \mathrm{L}$ (expressed per mole of monomer). The density of arrays is $2-5$ SWNTs/um. In some local areas (d) the density is up to $\sim 10 \mathrm{SWNTs} / \mathrm{\mu m}$. (e) The corresponding Raman spectrum. The peaks marked by blue triangles arised from the single-crystal quartz substrate. The excitation wavelength of the microRaman laser is $633 \mathrm{~nm}$. (f) AFM image were synthesized. In certain areas the density reached $\sim 10 \mathrm{SWNTs} / \mu \mathrm{m}$. The Raman spectrum shows the typical RBM peak of SWNTs at $138.1 \mathrm{~cm}^{-1}$, proving that SWNTs were obtained. Compared with the arrays of SWNTs obtained by the previous method, the density is lower and not very uniform, and the SWNTs show a little curvature in the AFM image (Fig. 3(f)).

\section{Discussion}

In both procedures, the presence of photoresist or PVP is the key for successfully synthesizing aligned arrays of SWNTs. Because the main components of Shipley 1827 photoresist are a mixed cresol novolak resin, $\sim 70 \%$ organic solvent, and $\sim 10 \%$ diazo photoactive compound (referring to the Material Safety Data Sheet (MSDS) data), we can consider this photoresist as an organic solution of the polymer (resin). The two polymers have three functions in our experiments.

First, ordered catalyst structures were easily and reproducibly patterned on quartz wafers by photolithography and PDMS stamp $\mu \mathrm{CP}$ techniques. Otherwise, $\mathrm{FeCl}_{3}$ as the catalytic precursor is hard to uniformly pattern on the surface of wafers, especially for high concentrations of $\mathrm{FeCl}_{3}$ [28]. Other catalysts such as ferritin $[29,30]$, FeMo clusters [31], and Fe/Mo [32] nanoparticles also cannot be directly patterned on a wafer surface. Rogers' group used photoresist lines as the mask and e-beam evaporation to obtain sub-nano Fe film patterns on quartz wafers $[7,18]$. However, we think the thickness of the Fe film is too thin to control by e-beam 
evaporation; furthermore our method of directly doping $\mathrm{FeCl}_{3}$ into the photoresist is much easier.

Secondly, the polymer layer can effectively prevent the formation of large particles during the drying step at room temperature and the heating step at 700 ${ }^{\circ} \mathrm{C}$. For $\mu \mathrm{CP}$ processes $\mathrm{Fe}_{x} \mathrm{O}_{y}$ particles arising from the hydrolysis of $\mathrm{FeCl}_{3} \cdot 6 \mathrm{H}_{2} \mathrm{O}$ easily agglomerate into bulk sizes on the PDMS stamp surface when the ink solution is dried in air. The formation of monodispersed catalyst nanoparticles is the prerequisite for catalyzing the CVD growth of SWNTs. Figure 4 shows uniform catalyst nanoparticle formation on the substrate after heating wafers at $700{ }^{\circ} \mathrm{C}$ in air for $5 \mathrm{~min}$. The average diameter of the nanoparticles was $\sim 6 \mathrm{~nm}$ when Shipley 1827 photoresist was used as the polymer layer (Fig. 4(a)). When $40 \mathrm{mmol} / \mathrm{L}$ PVP solution (expressed per mole of monomer) was applied, the diameters of most nanoparticles were less than $1 \mathrm{~nm}$ and the density of nanoparticles was higher than in the former case. Interestingly, in our experimental there appears to be no relationship between the sizes and density of nanoparticles and the quality of arrays of SWNTs they generated.

Finally, the polymer layer can help the substrates anchor the catalyst nanoparticles. We speculate that a residual amount of polymer is left on substrates when SWNTs begin to grow from catalyst lines. In AFM images (Figs. 2(b) and 3(f)) the substrates are seen to be very clean meaning that there is no diffusion of nanoparticles to the areas between catalyst lines. In order to confirm this speculation, we carried out a parallel experiment using different concentrations of PVP and without PVP. When there was no PVP present, large aggregative particles formed on the surface and many curved or random tubes were observed (Fig. 5(a)). With increasing concentration of PVP up to 20 $\mathrm{mmol} / \mathrm{L}$, the number of curved or random tubes gradually decreased, and the catalyst lines were more obvious after the growth of SWNTs and the arrays more aligned. However, too high a concentration of PVP will give rise to significant amounts of amorphous carbon which poisons the catalyst nanoparticles during the CVD process and when $40 \mathrm{mmol} / \mathrm{L}$ PVP solution was used, the density of arrays (shown in Fig. 5(d)) decreased. These results prove that the polymer layer can effectively retain catalyst particles on the substrates during the growth of SWNTs.

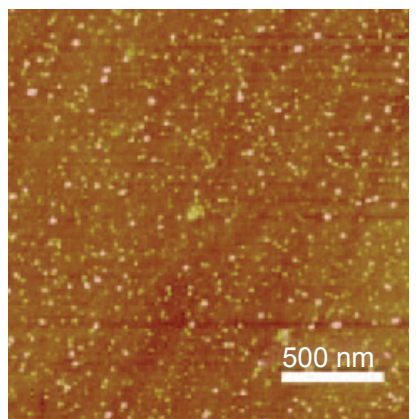

(a)

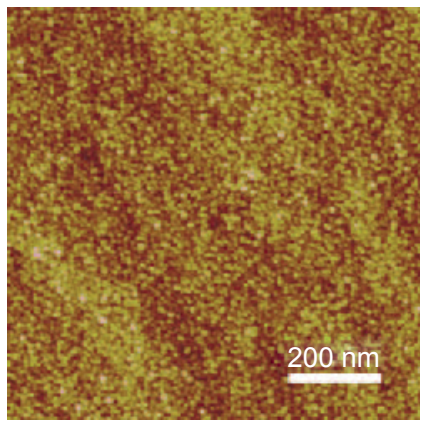

(b)
Figure 4 AFM images of catalyst nanoparticles formed by heating quartz wafers patterned with $\mathrm{FeCl}_{3} /$ Shipley 1827 photoresist (a) or $\mathrm{FeCl}_{3} / \mathrm{PVP}(\mathrm{b})$ at $700{ }^{\circ} \mathrm{C}$ for $5 \mathrm{~min}$

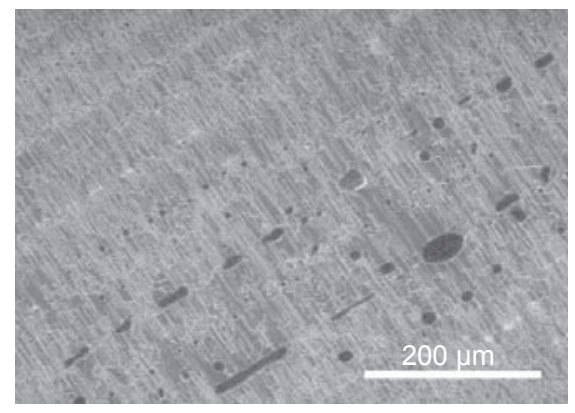

(a)

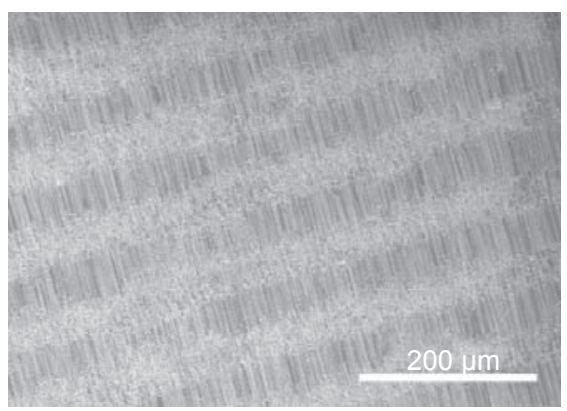

(c)

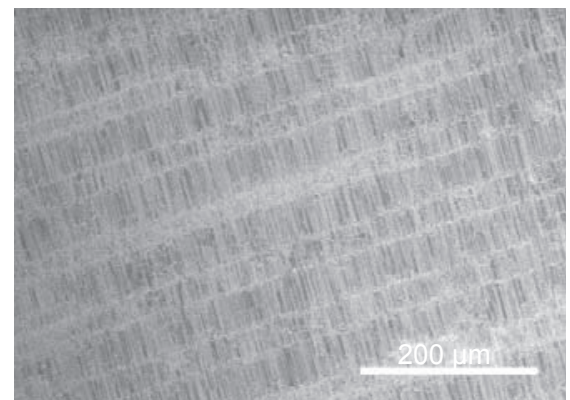

(b)

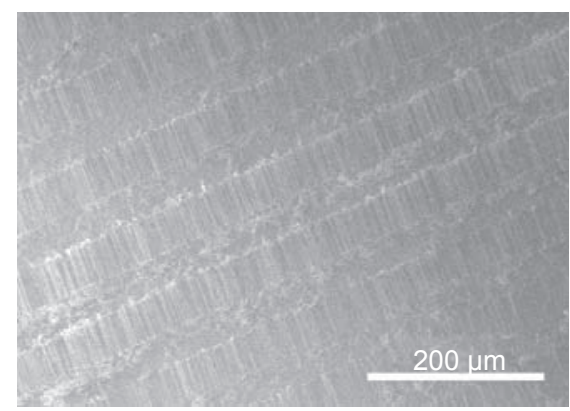

(d)

Figure 5 SEM images of the arrays of SWNTs grown on quartz wafers by using mixtures of 10 $\mathrm{mmol} / \mathrm{L} \mathrm{FeCl}_{3}$ and no PVP (a), $10 \mathrm{mmol} / \mathrm{L} \mathrm{PVP} \mathrm{(b),} 20 \mathrm{mmol} / \mathrm{L} \mathrm{PVP} \mathrm{(c),} \mathrm{and} 40 \mathrm{mmol} / \mathrm{L} \mathrm{PVP} \mathrm{(d),}$ as catalytic precursors. A reduction time of 10 min was employed prior to CVD growth of SWNTs 
Very recently, Ding et al. found that by using $\mathrm{CuCl}_{2} / \mathrm{PVP}$ as catalytic precursors and ethanol as the carbon feedstock, the resulting SWNT array density on quartz wafers was in excess of $50 \mathrm{SWNTs} / \mu \mathrm{m}$ in local areas [19]. We think that polymer assistance of CVD growth of SWNTs is a universal method and can be applied in various kinds of catalyst systems. It also seems to be the case that the small differences in array density are caused by use of different carbon feedstocks because the decomposition of ethanol is much easier than that of methane gas, and it has been shown that water, as a thermal decomposition product of ethanol, can contribute to removal of the amorphous carbon formed during the CVD growth of SWNTs [33]. Ding et al. found that the catalyst particles were preferentially retained at the tip of SWNTs and therefore presumed that SWNTs grew on quartz wafers by a tip-growth mechanism [19]. However, in our CVD results, we observed the existence of many nanoparticles only in the catalyst areas, and there were very few particles in the gaps between catalyst lines. We speculate this is caused by the different behavior of the two catalyst metals ( $\mathrm{Cu}$ and $\mathrm{Fe}$ ) [10], and we are more inclined to believe that the base-growth mechanism is at work in our experiments.

\section{Conclusions}

In summary, we present a universal and simple method of polymer assisted CVD growth of SWNTs arrays on quartz wafers. In our experiments, Shipley 1827 photoresist and PVP were used to pattern catalyst lines on quartz wafers by photolithography and PDMS $\mu \mathrm{CP}$ techniques. The polymers also contribute to the formation of catalyst nanoparticles and anchor the nanoparticles on the substrates. Patterned, dense, and high-quality arrays of SWNTs on a large scale were successfully synthesized by CVD of methane. Although our simple one-step catalyst preparation method has been demonstrated using Shipley 1827 photoresist on quartz, it should also be possible to use the same technique on any substrate (e.g., Si or sapphire), and to use it with other photoresists or electron-beam resists, such as PMMA or SU8. Further study of the growth mechanism and the fabrication of high-frequency devices based on the arrays of SWNTs are ongoing in our lab.

\section{Acknowledgments}

This work was supported by the Army Research Office and the Office of Naval Research. We thank Prof. Jie Liu's group at Duke University for providing assistance with AFM and Raman spectroscopy.

\section{References}

[1] Saito, R.; Dresselhaus, M. S.; Dresselhaus, G. Physical Properties of Carbon Nanotubes; World Scientific Publishing: Singapore, 1998.

[2] Anantram, M. P.; Leonard, F. Physics of carbon nanotube electronic devices. Rep. Prog. Phys. 2006, 507, 507-561.

[3] Kong, J.; Soh, H. T.; Cassell, A. M.; Quate, C. F.; Dai, H. J. Synthesis of individual single-walled carbon nanotubes on patterned silicon wafers. Nature 1998, 395, 878-881.

[4] Javey, A.; Guo, J.; Farmer, D. B.; Wang, Q.; Wang, D. W.; Gordon, R. G.; Lundstrom, M.; Dai, H. J. Carbon nanotube field-effect transistors with integrated ohmic contacts and high-k gate dielectrics. Nano Lett. 2004, 4, 447-450.

[5] Zhang, Z. Y.; Liang, X. L.; Wang, S.; Yao, K.; Hu, Y. F.; Zhu, Y. Z.; Chen, Q.; Zhou, W. W.; Li, Y.; Yao, Y. G.; Zhang, J.; Peng, L. M. Doping-free fabrication of carbon nanotube based ballistic CMOS devices and circuits. Nano Lett. 2007, 7, 3603-3607.

[6] Kocabas, C.; Hur, S. H.; Gaur, A.; Meitl, M. A.; Shim, M.; Rogers, J. A. Guided growth of large-scale, horizontally aligned arrays of single-walled carbon nanotubes and their use in thin-film transistors. Small 2005, 1, 11101116.

[7] Kang, S. J.; Kocabas, C.; Ozel, T.; Shim, M.; Pimparkar, N.; Alam, M. A.; Rotkin, S. V.; Rogers, J. A. High-performance electronics using dense, perfectly aligned arrays of singlewalled carbon nanotubes. Nat. Nanotechnol. 2007, 2, 230-236.

[8] Ismach, A.; Segev, L.; Wachtel, E.; Joselevich, E. Atomicstep-templated formation of single wall carbon nanotube patterns. Angew. Chem. Int. Ed. 2004, 43, 6140-6143.

[9] Han, S.; Liu, X.; Zhou, C. Template-free directional growth of single-walled carbon nanotubes on a- and r-plane sapphire. J. Am. Chem. Soc. 2005, 127, 5294-5295.

[10] Zhou, W. W.; Han, Z. Y.; Wang, J. Y.; Zhang, Y.; Jin, Z.; 
Sun, X.; Zhang, Y. W.; Yan, C. H.; Li, Y. Copper catalyzing growth of single-walled carbon nanotubes on substrates. Nano Lett. 2006, 6, 2987-2990.

[11] Burke, P. J. AC performance of nanoelectronics: Towards a ballistic $\mathrm{THz}$ nanotube transistor. Solid-State Electron. 2004, 48, 1981-1986.

[12] Zhen, Y; Burke, P. J. Aligned array FETs as a route towards $\mathrm{THz}$ nanotube transistors. Proc. SPIE-Int. Soc. Opt. Eng. 2005, 5790, 246-253.

[13] Guo, J.; Hasan, S.; Javey, A.; Bosman, G.; Lundstrom, M. Assessment of high-frequency performance potential of carbon nanotube transistors. IEEE Trans. Nanotechnol. 2005, 4, 715-721.

[14] Hasan, S.; Salahuddin, S.; Vaidyanathan, M.; Alam, A. A. High-frequency performance projections for ballistic carbon-nanotube transistors. IEEE Trans. Nanotechnol. 2006, 5, 14-22.

[15] Bethoux, J.-M.; Happy, H.; Derycke, G. D. V.; Goffman, M.; Bourgoin, J.-P. An 8-GHz fT carbon nanotube fieldeffect transistor for gigahertz range applications. IEEE Electron Device Lett. 2006, 27, 681-683.

[16] Akinwande, D.; Close, G. E.; Wong, H. S. P. Analysis of the frequency response of carbon nanotube transistors. IEEE Trans. Nanotechnol. 2006, 5, 599-605.

[17] Kocabas, C.; Kim, H.-s.; Banks, T.; Rogers, J. A.; Pesetski, A. A.; Baumgardner, J. E.; Krishnaswamy, S. V.; Zhang, $\mathrm{H}$. Radio frequency analog electronics based on carbon nanotube transistors. Proc. Natl. Acad. Sci. U.S.A. 2008, 105, 1405-1409.

[18] Kocabas, C.; Kang, S. J.; Ozel, T.; Shim, M.; Rogers, J. A. Improved synthesis of aligned arrays of single-walled carbon nanotubes and their implementation in thin film type transistors. J. Phys. Chem. C 2007, 111, 1787917886.

[19] Ding, L.; Yuan, D. N.; Liu, J. Growth of high-density parallel arrays of long single-walled carbon nanotubes on quartz substrates. J. Am. Chem. Soc. 2008, 130, 54285429.

[20] Kocabas, C.; Shim, M.; Rogers, J. A. Spatially selective guided growth of high-coverage arrays and random networks of single-walled carbon nanotubes and their integration into electronic devices. J. Am. Chem. Soc. 2006, 128, 4540-4541.

[21] Cassell, A. M.; Franklin, N. R.; Tombler, T. W.; Chan, E. M.; Han, J.; Dai, H. J. Directed growth of free-standing single-walled carbon nanotubes. J. Am. Chem. Soc.
1999, 121, 7975-7976.

[22] Huang, S. M.; Maynor, B.; Cai, X. Y.; Liu, J. Ultralong, well-aligned single-walled carbon nanotube architectures on surfaces. Adv. Mater. 2003, 15, 1651-1655.

[23] Zhen. Y.; Li, S. D.; Burke, P. J. Synthesis of aligned arrays of millimeter long, straight single walled carbon nanotubes. Chem. Mater. 2004, 16, 3414-3416.

[24] Li, S. D.; Zhen. Y.; Rutherglen, C.; Burke, P. J. Electrical properties of $0.4 \mathrm{~cm}$ long single walled carbon nanotubes. Nano Lett. 2004, 4, 2003-2007.

[25] Iler, R. K. The Chemistry of Silica: Solubility, Polymerization, Colloid and Surface Properties, and Biochemistry; Wiley: New York, 1979.

[26] Ago, H.; Imamoto, K.; Ishigami, N.; Ohdo, R.; Ikeda, K.; Tsuji, M. Competition and cooperation between latticeoriented growth and step-templated growth of aligned carbon nanotubes on sapphire. Appl. Phys. Lett. 2007, 90,123112 .

[27] Dresselhaus, M. S.; Dresselhaus, G.; Saito, R.; Jorio, A. Raman spectroscopy of carbon nanotubes. Phys. Rep. 2005, 409, 47-99.

[28] Ding, L.; Zhou, W. W.; Chu, H. B.; Zhong, L.; Zhang, Y.; $\mathrm{Li}, \mathrm{Y}$. Direct preparation and patterning of iron oxide nanoparticles via microcontact printing on silicon wafers for the growth of single-walled carbon nanotubes. Chem. Mater. 2006, 18, 4109-4114.

[29] Li, Y. M.; Kim, W.; Zhang, Y. G.; Rolandi, M.; Wang, D. W.; Dai, H. J. Growth of single-walled carbon nanotubes from discrete catalytic nanoparticles of various sizes. J. Phys. Chem. B 2001, 105, 11424-11431.

[30] Abrial, A.; Bouvier, J.; Renaudin, M.; Senn, P.; Vivet, P. A new contactless smart card IC using an on-chip antenna and an asynchronous microcontroller. IEEE J. Solid-State Circuits 2001, 36, 1101-1107.

[31] An, L.; Owens, J. M.; McNeil, L. E.; Liu, J. Synthesis of nearly uniform single-walled carbon nanotubes using identical metal-containing molecular nanoclusters as catalysts. J. Am. Chem. Soc. 2002, 124, 13688-13689.

[32] Li, Y.; Liu, J.; Wang, Y. Q.; Wang, Z. L. Preparation of monodispersed Fe-Mo nanoparticles as the catalyst for CVD synthesis of carbon nanotubes. Chem. Mater. 2001, 13, 1008-1014.

[33] Hata, K.; Futaba, D. N.; Mizuno, K.; Namai, T.; Yumura, M.; lijima, S. Water-assisted highly efficient synthesis of impurity-free single-walled carbon nanotubes. Science 2004, 306, 1362-1364.

\section{国备 Springer}

